\title{
Generación de posturas corporales en agentes virtuales a partir de evidencia neurocientífica
}

\author{
Diana G. Gómez-Martínez, Marco Ramos, Vianney Muñoz-Jiménez, \\ J. Raymundo Marcial-Romero \\ Universidad Autónoma del Estado de México, Toluca, Estado de México \\ México \\ dgomezm279@alumno.uaemex.mx, marco.corchado@gmail.com, \\ vmunozj@uaemex.mx, jrmarcialr@uaemex.mx
}

\begin{abstract}
Resumen. La Inteligencia Artificial busca proponer nuevos mecanismos de interacción humano-computadora con un alto mimetismo en comportamientos humanos. Por otro lado, la Neurociencia estudia los procesos cognitivos que están presentes en la generación de agentes autónomos, y que representan un desafió para mostrar comportamientos similares a los humanos para una interacción más natural. Es así, que en este artículo se propone un modelo para generar posturas corporales con respecto a estados emocionales en agentes virtuales basado en la neurociencia, que posteriormente en la interacción los usuarios sean capaces de reconocer y en consecuencia pueda ajustar su comportamiento propio para el logro de tareas y objetivos.
\end{abstract}

Palabras clave: agente virtual, postura corporal, neurociencia, inteligencia artificial.

\section{Body Postures Reproduction in Virtual Agents based on Neuroscience Evidence}

\begin{abstract}
Artificial Intelligence aims to propose new mechanisms of interaction between humans and computers with a high level of mimicry regarding human behavior. In another hand, neuroscience studies the cognitive processes that can be used to develop autonomous agents, and that represent the challenge to create one interaction between humancomputer the more natural possible. Hence in this paper proposes a model to reproduce body postures on emotional states on virtual agents based on the field Neuroscience, so that by the time interaction arrives users will be able to recognize and consequently adjust their behavior to achieve tasks and objectives.
\end{abstract}

Keywords: virtual agent, body posture, neuroscience, artificial intelligence. 


\section{Introducción}

Actualmente el uso de agentes virtuales en diferentes áreas de investigación tales como: medicina [5], psicología [11], enseñanza [24], comercio [1], etc. está creciendo sustancialmente, debido a que los agentes virtuales están desempeñando diversos mecanismos que buscan lograr una interacción transparente entre el usuario (ser humano) y la computadora [4]. Este proceso de interacción deberá asemejar la interacción que existe entre los humanos y lograr esto requiere conocer los mecanismos basados en neurociencias para comprender cómo la parte motora del cerebro es regulada por la evaluación emocional, que nos permita movimiento corporal en un agente virtual.

Los agentes virtuales deberán tener la capacidad de moverse tanto corporalmente como en su ambiente en el cual se encuentran embebidos, el movimiento corporal tiene alto impacto en el proceso de comunicación [7], ya que funge como medio para expresar información específica de estados emocionales a partir de variaciones de movimientos y de posturas del cuerpo, además la Psicología ha demostrado que el movimiento y las posturas corporales desempeñan un papel importante en el proceso de regulación emocional [26,27].

Cabe añadir que los agentes [30] virtuales están siendo utilizados en sistemas que tienen como objetivo apoyar en las tareas del ser humano, por ejemplo, los asistentes virtuales utilizados convencionalmente en la telefonía hasta avatares que intentan ayudar a personas con problemas de relación social (p. ej. autismo, esquizofrenia, entre otras) [2]. Por lo que ha sido necesario desarrollar modelos para la generación de agentes virtuales que simulen un ser humano que sea capaz de moverse y comunicarse como si se tratase de nosotros mismos.

Uno de los objetivos que persigue esta propuesta es generar agentes virtuales capaces de reproducir posturas corporales con respecto al estado emocional que desea manifestar, para que de este modo se logre una empatía con el usuario además de facilitar la interacción. Para ello este proceso que encierra la relación de un estado emocional con una postura corporal determinada se respaldó por un modelo cognitivo, dado que éste se basa en explorar la capacidad de las mentes humanas para modificar y controlar la forma en que los estímulos afectan la conducta, sustenta al aprendizaje como un proceso en donde se modifican significados de manera interna. Un modelo cognitivo representa una aproximación de los procesos cognitivos de un ser vivo, lo cual se hace con base en las distintas aproximaciones, una de estas aproximaciones es el enfoque de la neurociencia a partir del uso de los resultados encontrados en la Neurociencia Aplicada [28].

\section{Trabajos relacionados}

\subsection{Emociones}

La Psicología ha demostrado que la expresión de las emociones es representada mediante una postura corporal además del habla. Una postura corporal es entendida como una configuración de las articulaciones del cuerpo, para el análisis de estas configuraciones se precisan determinados parámetros con la 
finalidad de facilitar su descripción y distinguir una de otra. Por ejemplo, en el trabajo de Kleinsmith et al. [17] distinguen 24 características para definir una postura corporal, para la determinación de estas características se basaron en los tres planos ortogonales (sagital, horizontal y frontal), la medida de la extensión lateral, frontal y vertical del cuerpo, torsión del cuerpo, la inclinación de la cabeza y hombros, y en la orientación del cuerpo.

El trabajo de Kleinsmith et al. [15] partió de precisar las posturas corporales conforme las 6 emociones básicas (enojo, miedo, alegría, sorpresa, desagrado y tristeza) definidas por Ekman y Friesen [8], las posturas fueron mostradas a un conjunto de personas cuya cultura no era la misma con el propósito de identificar el impacto que tiene la cultura en la identificación de las emociones, de sus resultados derivaron que las posturas que tienden a confundirse son: el miedo con la sorpresa y el enojo con el desagrado, caso semejante pasa con las expresiones faciales [14].

Por otro lado, Shafir en sus estudios [26,27] ha mostrado que a partir de un conjunto de características motoras tales como el cuerpo, esfuerzo, forma y espacio (mismas que son estudiadas en Análisis de Movimiento Laban) se puede realizar la identificación de emociones específicamente la felicidad, enojo, miedo y tristeza, de las cuatro características dos de ellas se basan en la configuración del cuerpo tales son el cuerpo y el espacio.

En dichos estudios obtuvieron posturas corporales peculiares que son interpretadas en el marco de una emoción, con respecto al enojo los movimientos son rápidos, fuertes y directos, la cabeza ligeramente inclinada al frente, con los brazos estrechados en frente; el miedo fue predicho con un cuerpo condensado y cerrado, que conforme el espacio tendía a moverse hacia atrás, con el torso inclinado hacia atrás (es decir, con postura de retirada) y hombros de la misma forma. Para la felicidad los movimientos eran brincos rítmicos, con los brazos levantados que describían movimientos libres juntamente con un cuerpo expandido horizontalmente. Finalmente, con la tristeza el cuerpo se mostraba hundido con perdida de tono muscular, torso hundido y cabeza abajo.

Desde el punto de vista de Neurociencias las emociones son procesos complejos que tienen gran influencia en el comportamiento de las personas, que implican una respuesta motora tanto facial como todo el cuerpo, mismo que conlleva un proceso cognitivo que realiza la valoración de la situación [6] para generar una respuesta adecuada. El procesamiento emocional puede llevarse a nivel consciente e inconsciente, las conexiones que la amígdala mantiene con el hipotálamo producirán una respuesta emocional, sin que hasta el momento la información hubiera alcanzado la corteza y mediación consciente. En lo que concierne al aspecto consciente de las emociones, las estructuras involucradas incluyen la porción anterior del cíngulo, la corteza prefrontal orbital y ventromedial, el lóbulo temporal y la ínsula [21].

Cabe agregar que se han desarrollado modelos enfocados a generar el proceso emocional en agentes virtuales, tal es el caso de Rosales et al. [13] que proponen un modelo para la regulación emocional con memoria para agentes virtuales, en donde definen la regulación emocional como el proceso que valora el estado 
emocional y calcula el comportamiento apropiado para el logro de las metas. El modelo propuesto se fundamenta en evidencia neurocientífica y psicológica, mismo que está conformado por tres fases:

- Respuesta emocional: genera el comportamiento emocional visceral y la información procesada es enviada a la amígdala para calcular la emoción de esta información.

- Regulación emocional: basada en las técnicas de regulación emocional propuestas en el modelo de Gross [12], se recibe la decisión tomada y acorde a ésta se encuentra un comportamiento emocional apropiado, si la emoción calculada por la amígdala no es la adecuada el proceso de revaloración es activado y se calcula de nuevo la emoción.

- Comportamiento de salida: se concibe a partir del comportamiento emocional y el comportamiento de respuesta, este proceso tiene lugar en la corteza cingulada anterior.

\subsection{Sistema motor de control}

En el campo de la Neurociencia Aplicada [6] con base en estudios realizados han demostrado que el centro de locomoción del sistema motor está localizado en el lóbulo frontal [18] mismo que se encarga de planificar los movimientos, organizar la secuencia de movimientos y finalmente su ejecución (ver Figura 1).

El sistema motor está integrado jerárquicamente por varias estructuras que están interactuando llevando a cabo la transferencia de información aferente y eferente (ver Tabla 1) para llevar acabo el movimiento voluntario, es decir, aquel que está dirigido al cumplimiento de un objetivo, con la estructura jerárquica los diferentes niveles se influyen unos a otros, por lo que el flujo de información es tanto en serie (comunicación entre diferentes niveles) como en paralelo (múltiples vías entre cada nivel). En la Figura 2 se pueden identificar las estructuras principales que participan en la función motora, estructuras que se explican enseguida.

Tabla 1. Jerarquía del control motor [3].

\begin{tabular}{ccc}
\hline Nivel & Función & Estructuras \\
\hline Alto & Estrategia & Áreas asociadas a la neocortex, ganglio basal \\
Medio & Tácticas & Corteza motora, cerebelo \\
Bajo & Ejecución & Tronco cerebral, médula espinal \\
\hline
\end{tabular}


Generación de posturas corporales en agentes virtuales a partir de evidencia neurocientífica

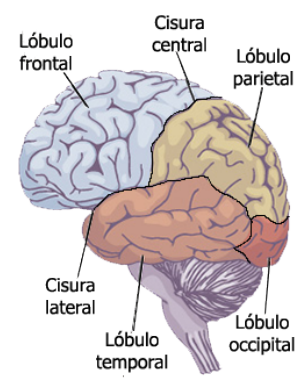

(a)

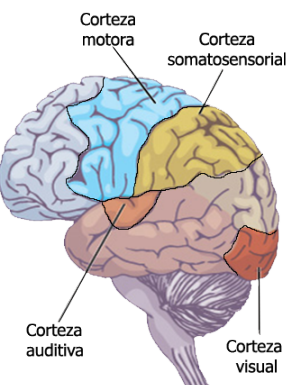

(b)

Fig. 1. a) Mapeo de los lóbulos en el cerebro [9]. b) Localización de la corteza motora en el lóbulo frontal [9].

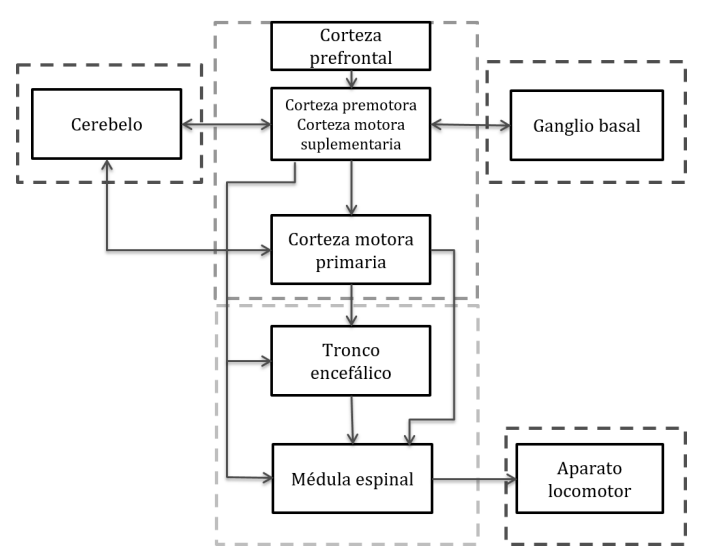

Fig. 2. Circuito del sistema motor [18].

- Ganglio basal. Facilita la selección e inhibición de movimientos y es la entrada para iniciar el movimiento.

- Cerebelo. Cumple la función de corregir errores en el movimiento comparando las ordenes motoras producidas con la retroalimentación sensorial sobre el movimiento que realmente se produjo, conjuntamente sincroniza los diferentes movimientos.

- Corteza premotora y Corteza motora suplementaria. La corteza premotora está involucrada en la selección de los planes motores apropiados para los movimientos voluntarios, también se encarga de seleccionar los programas motores basados en estímulos visuales, por otro parte la corteza motora suplementaria está asociada con escoger los movimientos basados en secuencias de movimientos recordados y en la práctica mental de estos movimientos. 
- Corteza motora primaria. Abarca la ejecución de movimientos seleccionados por la corteza premotora.

- Tronco encefálico y médula espinal. Todos los movimientos son producidos por neuronas motoras de la médula y el tronco encefálico que inervan los músculos. Estas motoneuronas están controladas y coordinadas por el encéfalo, por neuronas de la corteza cerebral y el tronco del encéfalo. Asimismo, la médula espinal aporta al control postural.

- Sistema locomotor. Está integrado por los músculos que al contraerse generan el movimiento de los huesos.

- Corteza parietal posterior. Procesa las relaciones espaciales de los objetos en el mundo y construye una representación del espacio externo que es independiente de la posición del ojo del observador.

- Corteza prefrontal. Realiza la evaluación de una actividad en particular.

Específicamente la corteza prefrontal y la corteza parietal posterior no son áreas motoras en sentido estricto, sin embargo, estas áreas son necesarias para asegurar que los movimientos se adapten a las necesidades del organismo y sean apropiados al contexto conductual.

Existen modelos encaminados a generar movimiento, uno de ellos fue propuesto por Shadmehr y Krakauer [25] el cual aportan que existen 3 situaciones que envuelven la generación de movimiento dirigido a un objetivo, estas situaciones son:

1. Sistema de identificación: la necesidad de predecir qué consecuencias sensoriales tendrá nuestro movimiento o comando motor.

2. Estado de estimación: realizar una combinación de las predicciones anteriores con la actual retroalimentación sensorial para crear una nueva creencia acerca del estado del cuerpo y el ambiente.

3. Control óptimo: con la creencia nueva del estado del cuerpo y del ambiente, se tiene que ajustar de nuevo el ciclo sensomotor de manera que maximice su desarrollo.

En 2009 Shadmehr y Krakauer [25] clasifican las siguientes estructuras cerebrales dirigidas a producir movimiento voluntario en donde cada una desempeña una tarea específica.

- Ganglio basal: provee el costo del comando motor y de los beneficios de la predicción del estado sensorial.

- Cerebelo: predice las consecuencias sensoriales del comando motor, es decir, provee los cambios propioceptivos y retroalimentación visual.

- Corteza parietal: combina la actual retroalimentación sensorial con la retroalimentación sensorial esperada, calculando una nueva creencia.

- Corteza motora y premotora: apoyándose de los costos motores y beneficios del estado sensorial previstos asignan una retroalimentación para el estado visual y propioceptivo (sentido que informa al organismo de la posición de los músculos), resultando un cambio a la creencia interna acerca del comando de motor. 
De manera semejante Madrigal et al. [19] en 2013, proponen un modelo para la generación de movimiento de una extremidad a partir de un músculo basado en una bio-arquitectura, cuyo objetivo consistió en replicar la forma en que la información eferente (información que viaja del cerebro al órgano receptor) y aferente (información que se transita del órgano receptor hacia el cerebro) es procesada entre las diversas regiones nerviosas produciendo el movimiento de una extremidad.

El modelo de Madrigal mantiene un enfoque jerárquico en el cual las estructuras superiores son responsables de las actividades complejas tales como la planeación y la coordinación de los movimientos sin tomar en cuenta el trabajo hecho por los músculos. Dicho modelo se integró por la corteza motora, ganglio basal, cerebelo, centros del tronco cerebral, neuronas del circuito local, pools de neuronas motoras, receptores sensoriales y músculo-esqueléticos. La implementación del modelo se centró en las estructuras inferiores, específicamente en el pool de neuronas motoras, con el fin de mostrar el movimiento continuo de una extremidad, mismo que es realizado por la activación y desactivación del pool de neuronas motoras. Las pruebas realizadas conducen en que la personificación del movimiento no todas las neuronas motoras pertenecientes al grupo son activadas, puesto que la activación depende del esfuerzo y la velocidad requerida para la realización del movimiento.

\section{Propuesta}

Esta propuesta está basada en un modelo cognitivo que permita la reproducción de posturas corporales a partir de la regulación emocional, en donde los agentes virtuales podrán adaptarse a las condiciones del ambiente. El modelo está fundamentado en evidencia neurocientífica y psicológica. De acuerdo con las definiciones en neurociencia se presenta el diagrama de conexión por el cual fluye la información como se ilustra en la Figura 3.

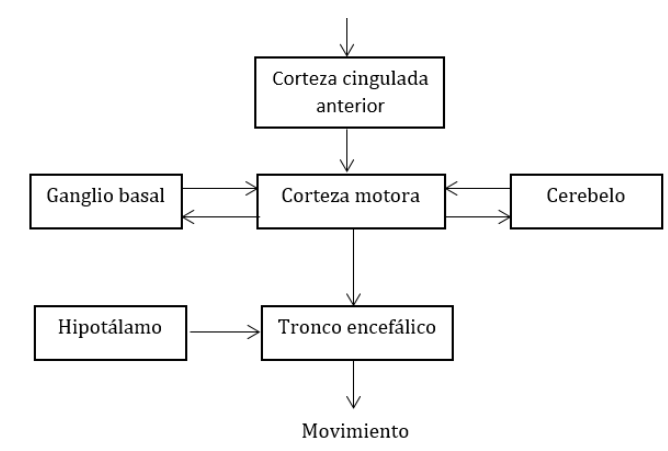

Fig. 3. Modelo cognitivo propuesto para generar posturas corporales con base en las emociones. 
El modelo expone como es que cada una de las estructuras del cerebro se van activando para poder generar una postura corporal a partir de la definición de una respuesta emocional con base en un estímulo. Este modelo está orientado a las estructuras superiores del circuito biológico (Figura 2) el cual aportan a la planificación, selección y coordinación de los movimientos.

El modelo parte de un estímulo que ocasionará una respuesta emocional, respuesta emocional que estará asociada a una emoción y a su vez la emoción a un plan con el fin de generar la respuesta motora (función que realiza la corteza cingulada anterior), para llevar a cabo dicho plan es necesario definir el conjunto de movimientos que son requeridos para ejecutar el plan, en seguida se le asignará la dirección y el esfuerzo de cada movimiento. Esta tarea es efectuada por la corteza motora, una vez que ya se tienen mapeados los movimientos es necesario fijar la secuencia de su ejecución.

Posteriormente esta secuencia de movimientos serán producidos por las estructuras inferiores del circuito biológico, dichos movimientos son generados conscientemente, ahora bien, si nos encausamos a las respuestas emocionales viscerales los cuales son inconscientes, el flujo de la información comienza del hipotálamo mismo que tiene una conexión con el tronco encefálico el cual realiza la inervación de los músculos para generar el movimiento, sin embargo antes no existió un plan el cual fuera asociado a los movimientos.

a) Planificación de la respuesta emocional

Para la obtención de las señales que representan la entrada a nuestro modelo serán basadas en el modelo cognitivo de Rosales et al. [22], modelo cuyo objetivo fue la generación de comportamientos apropiados en creaturas virtuales a partir de una regulación emocional, en donde el significado del estímulo $S$ es representado $M=\left\{E_{1}, E_{2}, E_{3}, E_{4}, E_{5}, E_{6}\right\}$ después de ser sometido a la regulación emocional, cada componente $M$ simboliza cada una de las seis emociones clasificadas por Ekman [8], cuyo valor es de tipo real en un intervalo $0 \leq E_{x} \leq 1$, personificando de esta manera la intensidad de cada emoción.

De este tipo son las señales recibidas en la estructura de la corteza cingulada anterior que posteriormente se evaluarán con el objetivo de definir a cuál de las emociones básicas tienden el conjunto de señales, o bien, como es que impactan las otras emociones. Para ello se realizará un análisis discriminatorio de las emociones a partir del establecimiento de umbrales de cada emoción.

Después de la determinación de la emoción, ésta será asociada a una postura corporal que representará el objetivo a llegar (dichas configuraciones serán basadas en los estudios de Kleinsmith et al. $[15,16]$ ), la asociación emoción-postura está apoyada por experiencias previas que van enriqueciendo el conocimiento y por consecuencia va moldeando la forma en que los seres humanos tendemos a expresarnos.

b) Mapeo de movimientos

Una vez que se tiene definido el objetivo es necesario definir qué partes del cuerpo participan, para ello se trabajará bajo un enfoque homuncular [29]. Es decir, nos apoyaremos en el homúnculo motor de Penfield que se refiere 
a un "hombrecillo en el cerebro" que hace referencia a la representación de los mapas corporales en el cerebro, mismo que indica la ubicación y la cantidad de corteza dedicada a cada parte del cuerpo. Las partes con mayor tamaño representa que sus movimientos son más complejos y requieren mayor precisión (por ejemplo, la mano, la boca y los pies), entonces la cantidad de neuronas motoras que estén alojadas en cada parte del cuerpo para la generación del movimiento deberá ser proporcional a su tamaño.

c) Codificación de la dirección del movimiento

Ahora bien, con respecto a la definición de la dirección del movimiento de las diferentes partes del cuerpo participes para generar la postura, basándose en la literatura $[3,10,20]$ en donde se menciona que la definición de la dirección de los movimientos está determinada por un conjunto de neuronas. Cada una de ellas responde durante el movimiento en cada dirección, la actividad de cada neurona es representada como un vector de dirección en donde cada neurona apunta en la dirección que mejor convenga en la celda (Figura 4a), y la longitud del vector representa el grado de actividad de esa neurona durante el movimiento.

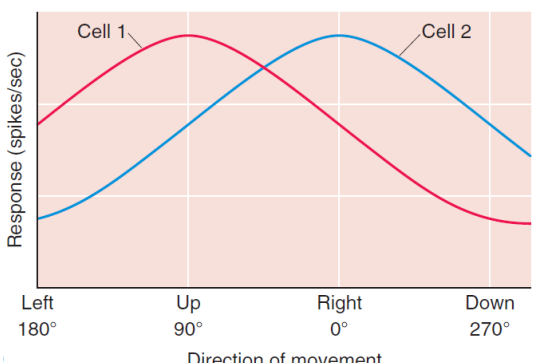

(a)

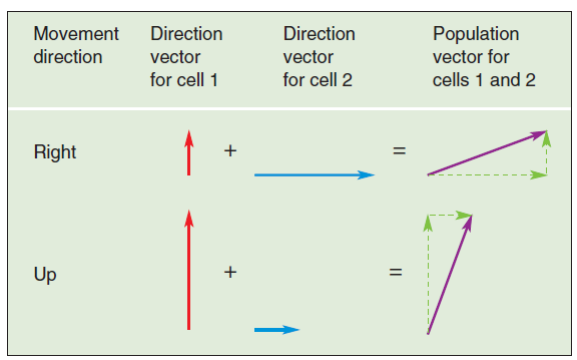

(b)

Fig. 4. a) Curvas de ajuste para 2 celdas en la corteza motora [3]. b) Respuesta de cada celda representada como un vector de dirección [3].

Los vectores que representan la actividad de cada neurona se trazarán juntos para cada dirección de movimiento (Figura 4b), luego se promediarán para producir un vector de población, que será representado por la ecuación 1, el cual estima la dirección del movimiento en un tiempo $(t)$ de una población de vectores de dirección $P(t)$, cuyo rendimiento es en cada celda $i$ en la dirección preferida $C_{i}$ ponderada por la actividad neuronal de la celda $V_{i}$ :

$$
P(t)=\sum_{i} V_{i}(t) C_{i}
$$

La relación entre la tasa de descarga de la neurona y la dirección del movimiento se describirá por una función de ajuste de coseno dada por la ecuación 
2 , donde $b_{i}$ y $a_{i}$ son los coeficientes de regresión y $\theta-\theta_{i}$ es el ángulo formado por la dirección preferida de la celda y la dirección del movimiento actual $M$. Cada parte del cuerpo anteriormente mapeada tendrá un vector de población asociado:

$$
V_{i}(M)=b_{i}+a_{i} * \cos \left(\theta-\theta_{i}\right) .
$$

d) Selección de la secuencia de movimientos

Una vez que se tienen las partes del cuerpo con su vector de población definido, se puntualizará la secuencia bajo la cual se ejecutarán los movimientos, para eso las diferentes partes del cuerpo estarán subagrupadas para generar movimiento en extremidades superiores e inferiores, tronco y cabeza [23]. A cada integrante del subgrupo se le ligará un peso el cual estará generado bajo la jerarquía de las articulaciones, con ello se poseerá un mapa de movimiento para ejecutarlo con las estructuras inferiores que participan en la función motora.

La Figura 5 muestra el esqueleto asociado al agente que deberá buscar la postura corporal adecuada mediante la respuesta emocional a partir de la percepción del ambiente.

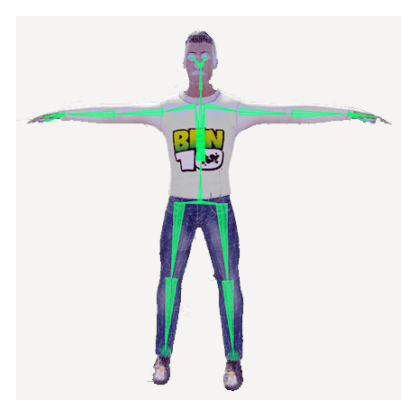

(a)

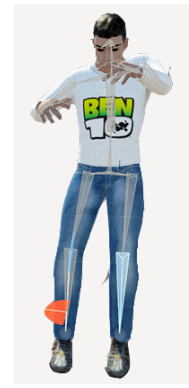

(b)

Fig. 5. a) Planificación de la respuesta emocional, mapeo de movimientos, codificación del esfuerzo y dirección del movimiento. b) Selección de la secuencia de movimientos.

\subsection{Resultados preliminares}

Los resultados obtenidos hasta este momento permiten observar el tratamiento de información aferente que se realiza en cada estructura cerebral, la cual está generando que el agente virtual adopte una postura corporal. Actualmente se trabajan con cuatro emociones: alegría, tristeza, miedo y enojo, se eligieron estas cuatro emociones ya que son la base de comparación con resultados de trabajos anteriormente citados, además la posibilidad de confundir una emoción 
con respecto a otra a partir de la postura corporal es remota, en otras palabras, hay una mayor facilidad de discriminar una emoción con relación a otra pues el umbral para el discernimiento es considerable.

Es necesario validar que el agente virtual adquiera las posturas corporales adecuadas con respecto a su estado emocional actual, mismo que es inducido por una retroalimentación del ambiente y que permita trasmitir este lenguaje al usuario y a otros agentes con los que comparte el ambiente, como se puede apreciar en las Figuras 6a, 6b, 6c y 6d. Para ello será necesario embeber al agente en un ambiente virtual, en donde el usuario tenga la experiencia de interactuar con el agente, el agente se apoyará de las posturas corporales para enfatizar el dialogo que se establezca. Es importante destacar que la postura corporal que exprese una emoción no debe ser siempre la misma pues puede existir un marco de variación de acuerdo a la secuencia de movimientos musculares y a la amplitud del ángulo entre las articulaciones, esta variación estará a juicio del usuario pues es quien discriminará la emoción que el agente pretende expresar.

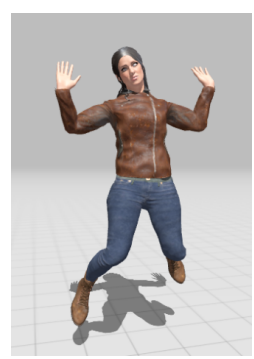

(a) Alegría

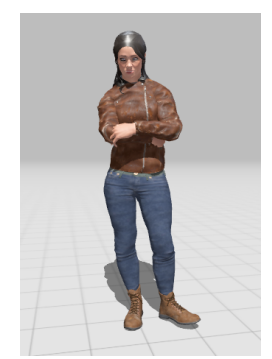

(b) Enojo

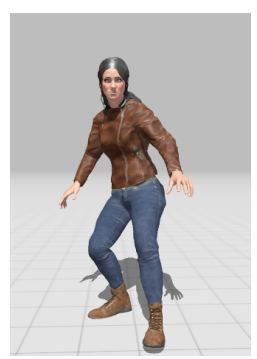

(c) Miedo

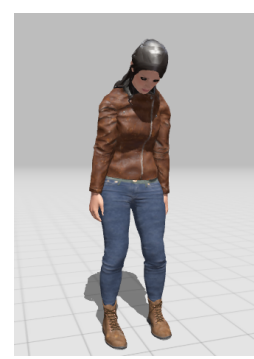

(d) Tristeza

Fig. 6. Posturas corporales a partir de una respuesta emocional.

Para poder definir que una postura es semejante a otra nos apoyaremos de la definición de una distancia máxima que podrá variar con respecto a la postura objetivo, esta distancia se obtendrá de las articulaciones que enfatizan la postura corporal.

\section{Conclusiones y trabajos futuros}

La integración de posturas corporales en agentes virtuales brindará mayor credibilidad a la expresión de sus estados emocionales además de atraer por un mayor intervalo de tiempo la atención del usuario, de esta manera se estará reforzando el proceso de interacción que existe entre el usuario y la computadora.

De igual manera, la integración del modelo cognitivo fundamentado en evidencia neurocientífica y psicológica permite que el proceso interno que se lleva acabo para producir una postura sea semejante al del ser humano, y no solo la respuesta externa que el usuario percibe, representando un respaldo para 
el comportamiento emocional, dado que ayudará a entender el porqué de la respuesta motora.

Es necesario la integración de parámetros que describan la personalidad del agente, personalidad que influirá en la evaluación del estímulo para determinar qué emoción la está produciendo, ya que experiencias previas representarán el detonante para la formación de la personalidad. También la incorporación de contextos bajo los cuales los agentes virtuales estarán inmersos para efectuar la ampliación del marco de las emociones, apoyará al discernimiento de las emociones conforme la postura que adopte el agente, reduciendo de esta manera el porcentaje de confusión entre las emociones sorpresa-miedo y enojo-desagrado.

\section{Referencias}

1. AIVO: Agentbot (2016), http://agentbot.net/es, accedido el 16-01-2017

2. AlterEgo: Proyecto alterego (2012), http://www . euromov. eu/alterego/, accedido el 02-02-2017

3. Bear, M.F., Conners, B.W., Paradiso, M.A.: Neurocience exploring the Brain. Lippincott Williams \& Wilkins, United States of America, third edn. (2007)

4. Bevacqua, E., Richard, R., Soler, J., Loor, P.D.: INGREDIBLE: A platform for full body interaction between human and virtual agent that improves co-presence. Archive ouverte HAL (Jul 2016)

5. Caballero Lambert, A.O., Torres Montañez, C.H.: Asistente virtual para el diagnóstico de trastornos alimentarios usando un motor cognoscitivo general: Nutmin. In: iaedp Symposium (2016)

6. Cardinali, D.P.: Neurociencia aplicada. Sus fundamentos. Editorial medica panamericana, Buenos Aires (2007)

7. Dael, N., Mortillaro, M., Scherer, K.R.: Emotion Expression in Body Action and Posture. American Psychological Association 12(5), 1085-1101 (2012)

8. Ekman, P., Friesen, W.V.: Unmasking the Face: A Guide to Recognizing Emotions from Facial Expressions. Malor Books (2015)

9. EMAZE: Sistema nervioso (Dec 2014), https://www.emaze.com/@AOIILCFI/ sistema-nervioso-pa, accedido el 28-03-2017

10. Georgopoulos, A.P., Schwartz, A.B., Kettner, R.E.: Neurona population coding of movement direction. Science 233, 1416-1419 (Sep 1986)

11. Gómez Cancino, C.K.: Psiquiatras virtuales para tratamiento remoto de pacientes con depresión (Aug 2016), http://www. conacytprensa.mx/index.php/ciencia/salud/ 9796-psiquiatras-virtuales-para-tratamiento-remoto-de-pacientes-con-depresion, accedido el 07-01-2017

12. Gross, J.: The emerging field of emotion regulation: An integrative review. Peer Reviewed Journal 2(3), 271-299 (1998)

13. Hernando Rosales, J., Jaime, K., Ramos, F., Ramos, M.: An Emotional Regulation Model with Memories for Virtual Agents. In: 12th IEEE International Conference on Cognitive Informatics \& Cognitive Computing (2013)

14. Jack, R.E., Garrod, O.G.B., Schyns, P.G.: Dynamic facial expressions of emotion transmit an evolving hierarchy of signals over time. Current Biology, Elsevier 24, 187-192 (Jan 2014) 
15. Kleinsmith, A., Bianchi-Berthouze, N., Silva, P.R.D.: Cross-Cultural Differences in Recognizing Affect from Body Posture. Elsevier Science 18(6), 1371-1389 (Dec 2006)

16. Kleinsmith, A., Bianchi-Berthouze, N., Steed, A.: Automatic Recognition of NonActed Affective Postures. IEEE Transactions on Systems, MAN, And CyberneticsPart B: Cybernetics 41(4), 1027-1038 (Aug 2011)

17. Kleinsmith, A., De Silva, P.R., Bianchi-Berthouze, N.: Grounding Affective Dimensions into Posture Features. Springer 3784, 263-270 (2005)

18. Kolb, B., Whishaw, I.Q.: Neuropsicología humana. Editorial medica panamericana, Argentina, quinta edn. (2006)

19. Madrigal, D., Torres, G., Del Valle, J.L., Ramos, F.: A Bio-Inspired Architecture of a Motor Neuron System for Virtual Creatures: Movement of a Single Limb with a Single Muscle. Procedia Computer Science 22, 440-449 (2013)

20. The National Academy of Sciences of the USA: Mapping of the preferred direction in the motor cortex, vol. 104 (Jun 2007)

21. Ostrosky, F., Vélez, A.: Neurobiología de las emociones. Revista Neuropsicología, Neuropsiquiatría y Neurociencias 13(1), 1-13 (Jan 2013)

22. Rosales, J.H., Ramos, F., Ramos, M.: Search for an appropiate behaviors within the emotional regulation in virtual creatures using learning classifier (2017)

23. Rosell Puig, W., González Fano, B., Dovale Borjas, C., Domínguez Hernández, L.: División regional del cuerpo humano para facilitar su estudio. Diferencias entre las regiones superficiales y esqueléticas. Educación Médica Superior 20(3) (Jul 2006)

24. Róspide, C.G., Puente, C.: Agentes virtuales inteligentes aplicados a un entorno educativo. Pensamiento matemático 2(2), 195-208 (Sep 2012)

25. Shadmehr, R., Krakauer, J.W.: The cognitive neurosciences, chap. V.Motor Systems. Computational neuroanatomy of voluntary motor control, pp. 587-597. Massachusetts Institute of Technology, fourth edition edn. (2009)

26. Shafir, T.: Using movement to regulate emotion: Neurophysiological findings and their application in psychotherapy. Frontiers in Psichology (Sep 2016)

27. Shafir, T., Tsachor, R.P., Welch, K.B.: Emotion regulation through movement: Unique sets of movement characteristics are associated with and enhance basic emotions. Frontiers in Psichology (Jun 2016)

28. Torres Blanco, G.A.: Metodología para el desarrollo de arquitecturas cognitivas basada en neurociencias. Ph.D. thesis, Cinvestav del IPN Unidad Guadalajara, México (2013)

29. Won, A.S., Bailenson, J., Lee, J., Lanier, J.: Homuncular flexibility in virtual reality. Journal of Computer-Mediated Communication 20, 241-259 (May 2015)

30. Wooldridge, M.: An Introduction to MultiAgent Systems. UK: John Wiley \& Sons, $\operatorname{ltd}(2009)$ 